九州大学学術情報リポジトリ

Kyushu University Institutional Repository

\title{
Bathymetric effects and corrections in marine CSEM data
}

\section{Sasaki, Yutaka}

Kyushu University, Department of Earth Resources Engineering

http://hdl. handle. net/2324/25553

出版情報: Geophysics. 76 (3)，pp.F139-F146，2011-04-12. Society of Exploration Geophysicists バージョン:

権利関係: (C) 2011 Society of Exploration Geophysicists. 


\title{
Bathymetric effects and corrections in marine CSEM data
}

\author{
Yutaka Sasaki ${ }^{1}$
}

\begin{abstract}
The effect of seafloor topographic variations on controlled-source electromagnetic (CSEM) responses is investigated using a finite-difference modeling approach for both petroleum and gas-hydrate exploration scenarios. In a deepwater reservoir model, distortions in the inline electric field responses are mainly due to galvanic effects, particularly at long transmitter-receiver (Tx-Rx) offsets, and are characterized by persistent short-wavelength anomalies at all Tx-Rx offsets in the amplitude profiles. In a shallow-water reservoir model, the contribution of inductive effects to bathymetric distortions is large at short Tx-Rx offsets compared to the deepwater case, whereas at long offsets, the distortion pattern is mainly determined by the source-receiver geometry
\end{abstract}

relative to the sea surface because of the airwave effect rather than by the resistivity variations associated with the seafloor topography. A simple correction technique, which is an extension of the topographic correction procedure for magnetotelluric data, is effective in removing the bathymetric distortions for the deep marine case, but cannot be used for the shallow-water case because of the airwave effect. For a gas-hydrate exploration model, it is shown that the bathymetric response can be large enough to mask the target response, because higher frequencies and shorter offsets need to be used to detect the shallow targets. However, the modeling study suggests that the shallow target response can be separated from the bathymetric distortions using the correction method.

\section{INTRODUCTION}

The marine controlled-source electromagnetic (CSEM) method is becoming an important tool for both offshore hydrocarbon exploration and gas-hydrate identification (e.g., Ellingsrud et al., 2002; Weitemeyer et al., 2006; Constable and Srnka, 2007). With increasing commercial applications of marine CSEM surveys, there has been considerable interest in hydrocarbon detection in more complex settings. Issues that may pose challenges include the shallow-water problem, bathymetry variations, near-surface variations of resistivity, anisotropy, and targets close to resistive basements.

The importance of bathymetric effects is well understood in the exploration industry (e.g., Darnet et al., 2007). These effects often have been simulated using finite-difference (FD) or finiteelement (FE) methods. Um (2005) examined the effect of a vertical cliff on the marine CSEM response using a 3D FD code and suggested that accurate bathymetry data should be collected for any marine CSEM survey. Using a 3D FD code, Hoversten et al. (2006) showed false anomalies produced by a gentle seafloor slope when data are normalized by 1D model responses.
Li and Constable (2007) implemented a new FE modeling algorithm that allows precise representation of 2D bathymetry with the use of the unstructured triangular mesh. Commer and Newman (2007) presented a synthetic example of the 3D inversion of marine CSEM data that incorporates seafloor topographic variations. Endo et al. (2008) proposed an integral equation modeling approach that handles bathymetry as an inhomogeneous background domain.

Ideally, seafloor topography should be incorporated into 3D inversion schemes as shown by Commer and Newman (2007), but actual applications of such inversions to real data are not common. Instead, a simple normalization procedure has been used in routine CSEM data analyses as a practical tool for identification of areas with responses that are anomalous when compared to the general background trend (Hesthammer et al., 2010). In this case, bathymetric effects can be accounted for by normalizing the CSEM data by the simulated response from a background bathymetric model, but the normalized response can be problematic unless the background resistivity is adequately determined. It is therefore instructive to show the extent to 
which seafloor topographic features can hamper identification of the target response for different hydrocarbon exploration scenarios and to see if a simple correction procedure can be used to separate the target signatures.

In this paper, a simple technique is presented that could be used to correct inline electric field data for bathymetric effects in some marine environments. Next, the accuracy of a 3D FD code (Sasaki and Meju, 2009) is validated for modeling seafloor topography by comparisons with the $2 \mathrm{D} \mathrm{FE}$ solution of $\mathrm{Li}$ and Constable (2007). Finally, simulated results for the detection of a petroleum reservoir and gas hydrate accumulations are presented to explain how galvanic, inductive, and airwave effects relate to bathymetric responses and to illustrate the effectiveness and limitations of the correction method.

\section{CORRECTION METHOD}

The topographic correction is not new to resistivity and magnetotelluric surveys. Fox et al. (1980) showed that DC resistivity data can be corrected for topographic effects by normalizing the observed data with the topographic model response and then by multiplying it by the theoretical response of a half-space. A similar procedure is used for magnetotelluric impedance,

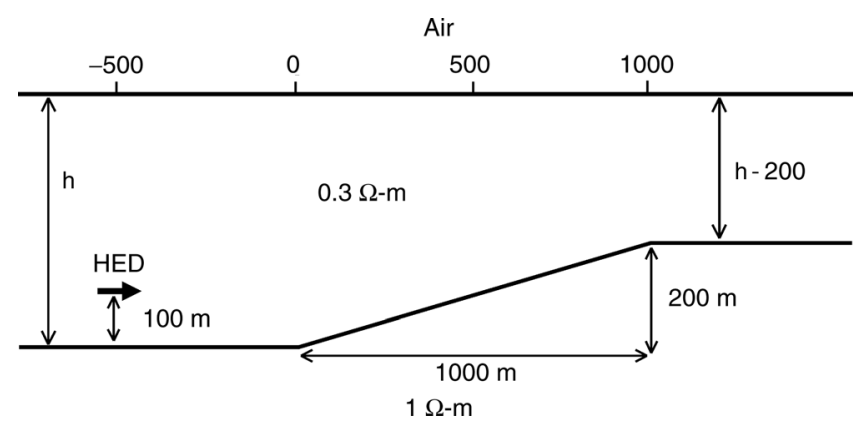

Figure 1. Seafloor ramp model used to test the accuracy of the FD solution.

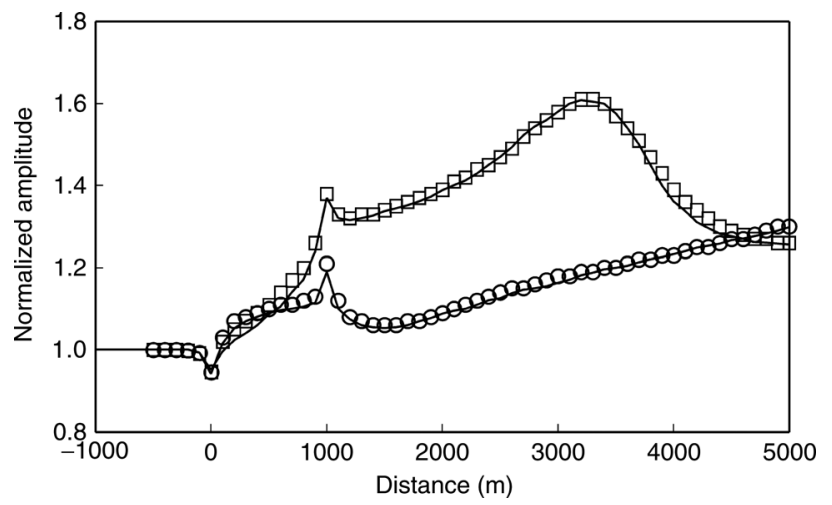

Figure 2. Comparisons of the FD (symbols) and FE (solid line) solutions for the inline horizontal electric field for the model shown in Figure 1, with two different water depths $(h=400$ and $1200 \mathrm{~m}$ ). The squares and circles are for the water depths $h=400$ and $1200 \mathrm{~m}$, respectively. The FE solution is redrawn from Figure $5 \mathrm{f}$ in $\mathrm{Li}$ and Constable (2007). but involves complex correction coefficients (Chouteau and Bouchard, 1988).

Here, this technique is extended to marine CSEM surveys to remove the bathymetric distortions from inline electric field responses. It assumes that the ratio of the bathymetrically distorted subsurface response to the bathymetric model response is equal to the ratio of the undistorted (or flat-seafloor) subsurface response to the theoretical response of a homogeneous half-space (underlying the seawater layer). That is,

$$
E_{D} / E_{B}=E_{U} / E_{0},
$$

where $E_{D}$ and $E_{U}$ are, respectively, the distorted and undistorted electric fields for a given source-receiver combination, and $E_{B}$ and $E_{0}$ are the calculated response for the bathymetric and halfspace models with the same resistivity, respectively. The undisturbed (or corrected) CSEM response can thus be obtained from

$$
E_{U}=\left(E_{D} / E_{B}\right) E_{0},
$$

or

$$
E_{U}=C E_{D}
$$

where $C$ is the complex correction coefficient given by

$$
C=E_{0} / E_{B} .
$$

\section{VERIFICATION OF 3D FD SOLUTION}

The accuracy of a FD modeling approach was verified for the seafloor topography model shown in Figure 1. The model involves a ramp on the seafloor with a height of $200 \mathrm{~m}$ and a horizontal length of $1000 \mathrm{~m}$. The seawater varies in depth from $h$ at the bottom of the ramp to $h-200$ at the top of the ramp. The resistivities of the seawater and sediment are assumed to be 0.3 and $1 \Omega-\mathrm{m}$, respectively. A horizontal electric dipole (HED) is located $500 \mathrm{~m}$ away horizontally from the lower edge of the ramp at a height of $100 \mathrm{~m}$ above the seafloor. The source frequency is $0.25 \mathrm{~Hz}$.

In the FD model, the earth and air were divided into a $170 \times 54 \times 44$ cell grid in the $x, y$, and $z$ directions. The smallest cells $(50 \times 100 \times 20 \mathrm{~m})$ were used to approximate the slope on the seafloor as discontinuous steps. The largest cells $(3.2 \times 3.2 \times$ $3.2 \mathrm{~km}$ ) were used at the corners of the model. The modeling code uses the secondary-field formulation to avoid problems associated with rapidly varying fields in the vicinity of the source. The primary field used in this calculation was the electric field for a layered (1D) half-space with a water depth $h$. Thus, the sediment region above the level corresponding to the bottom of the ramp constitutes the zone of anomalous resistivity, or equivalent source (Newman and Alumbaugh, 1995).

Figure 2 shows a comparison of the normalized amplitude of the inline horizontal electric field for two different seawater depths $(h=400$ and $1200 \mathrm{~m})$. The normalizing field is the primary field, that is, the electric field at the actual receiver points calculated for the 1D background model. As shown in Figure 2, the computed electric field is in good agreement with the FE solution of Li and Constable (2007). Figure 3 shows a comparison of the amplitudes of the magnetic field for the orthogonal direction for the two water depths, normalized by the corresponding response of the flat-seafloor background model. It is seen in Figure 3 that the comparison between the FD and FE 
solutions for the magnetic field responses is also good. In what follows, only the electric field responses, which are commonly used in routine CSEM surveys, are presented.

\section{EFFECT OF BATHYMETRY ON PETROLEUM RESERVOIR RESPONSE}

To investigate the effect of seafloor topography on the CSEM response of a 3D hydrocarbon reservoir, I consider a submarine ridge model (Figure 4). The central part of the seafloor is characterized by a trapezoidal rise that is 400-m high, 1000-m wide at the top and 5000-m wide at the base. I further consider deepwater and shallow-water cases, where the water depth at the bottom of the hill, $h$ is set to 2000 and $600 \mathrm{~m}$, respectively. The water has a resistivity of $0.3 \Omega-\mathrm{m}$, and the structure below the seafloor consists of a $100 \Omega-\mathrm{m}$ reservoir embedded in a ho-

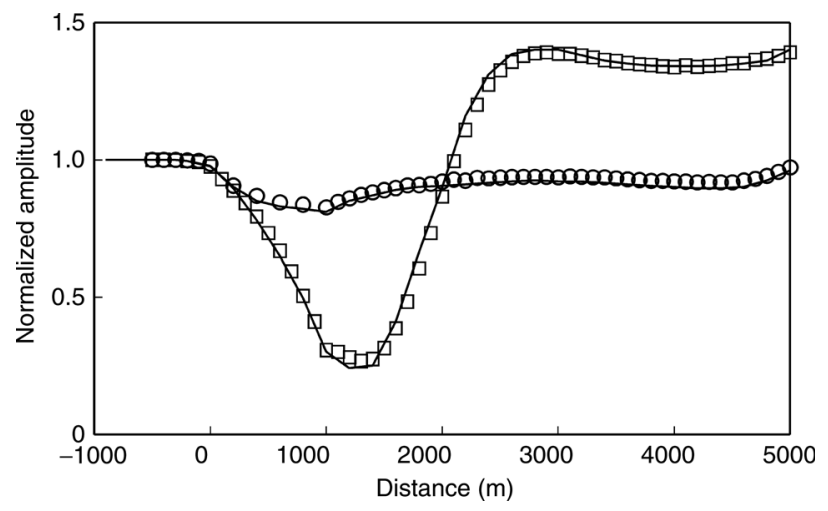

Figure 3. Comparisons of the FD (symbols) and FE (solid line) solutions for the orthogonal horizontal magnetic field for the model shown in Figure 1, with two different water depths $(h=400$ and $1200 \mathrm{~m})$. The squares and circles are for the water depths $h=400$ and $1200 \mathrm{~m}$, respectively. The FE solution is redrawn from Figure $7 f$ in $\mathrm{Li}$ and Constable (2007).

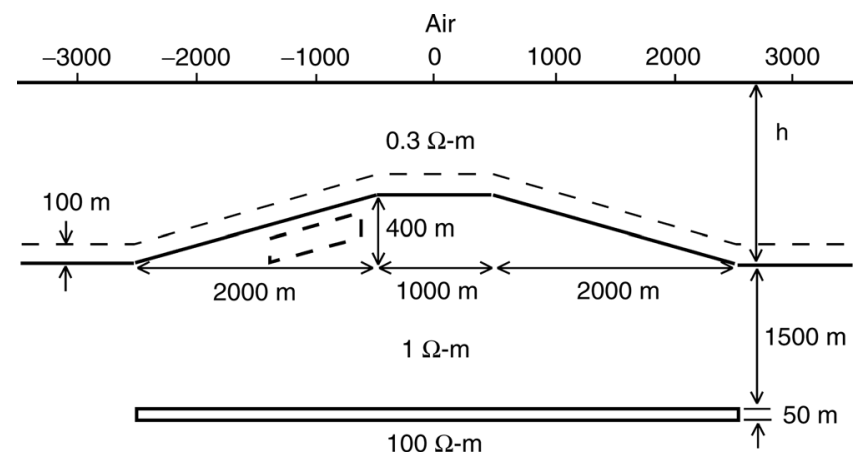

Figure 4. 3D hydrocarbon reservoir model with trapezoidal ridgelike seafloor topography. The HED is assumed to remain $100 \mathrm{~m}$ above the seafloor. Two water depths $(h=2000$ and $600 \mathrm{~m})$ are considered. The dashed parallelogram represents a shallow heterogeneity with a resistivity of $5 \Omega-\mathrm{m}$ at a burial depth of $80 \mathrm{~m}$, which is considered later to demonstrate the applicability of the bathymetric correction. Its thickness is $120 \mathrm{~m}$ and the horizontal extent is $800 \mathrm{~m}$ (from $x=-1400$ to $-600 \mathrm{~m}$ ). mogeneous background sediment with a resistivity of $1 \Omega-\mathrm{m}$. The reservoir has a lateral extent of $5000 \times 5000 \mathrm{~m}$ and thickness of 50. Its burial depth below the seafloor varies from 1500 to 1900 . A single profile with 43 HED sources of length 100 is assumed to run through the center of the reservoir $(x, y=0 \mathrm{~km})$ along the $x$-direction. The sources are also assumed to operate at $0.25 \mathrm{~Hz}, 100 \mathrm{~m}$ above the seafloor. The receivers are positioned every $200 \mathrm{~m}$ on the seafloor from $x=-8.5$ to $8.5 \mathrm{~km}$. The grid that was used has $189 \times 78 \times 52$ cells with a minimum cell size of $100 \times 100 \times 40 \mathrm{~m}$ in the region involving bathymetry and a maximum size of $6.4 \times 6.4 \times 6.4 \mathrm{~km}$ at the outer corners. For each source location, the primary field used was for a 1D model with a water depth coincident with the actual depth just below the source. To check the self-consistency of the model, the responses were also calculated using the primary field for a background model with the water depth fixed at the bottom of the ridge. The results obtained from both approaches are indistinguishable.

\section{Electric field characteristics in deepwater}

The inline horizontal electric field responses were first simulated for the deepwater models with and without the reservoir. In Figure 5, the amplitude and phase responses at selected transmitter-receiver (Tx-Rx) separations (1 to $7 \mathrm{~km}$ ) have been plotted as a function of Tx-Rx midpoint. Here, only the responses for the geometries where the source is located on the left side of the receiver are shown. From Figure 5a and b, one can see that the bathymetric distortion in the amplitude response occurs mainly where the source or receiver is located near discontinuities in slope, and is characterized by short-wavelength anomalies persisting at all Tx-Rx offsets while the distortion in the phase response show gentle variations particularly at larger offsets. This suggests that like the distortions from small-scale,

a)

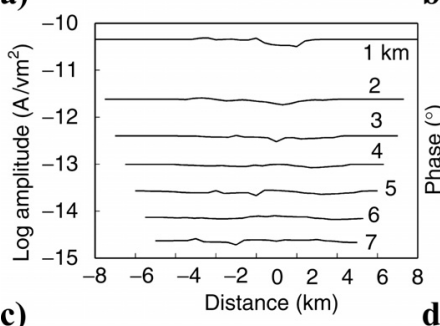

b)
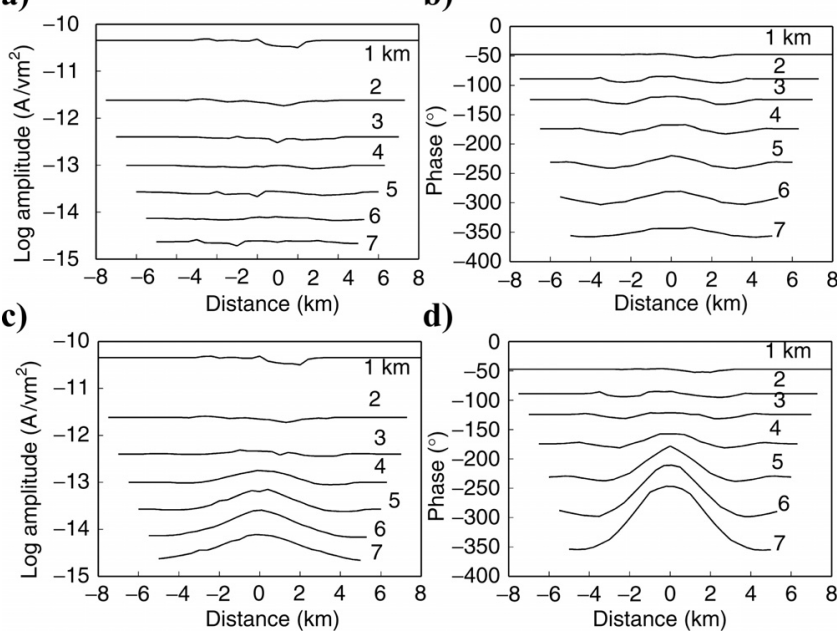

Figure 5. Inline horizontal electric field responses at selected Tx$\mathrm{Rx}$ offsets (1 to $7 \mathrm{~km}$ ), plotted as a function of the Tx-Rx midpoint for the deepwater bathymetric models with and without the resistive reservoir ( $h=2000 \mathrm{~m}$ in Figure 4). (a) Amplitude and (b) phase for the model without the reservoir; (c) amplitude and (d) phase for the model with the reservoir. The source frequency is $0.25 \mathrm{~Hz}$. 
shallow heterogeneities (Sasaki and Meju, 2009), the bathymetric distortion of the electric field in this deepwater reservoir model is mainly the result of the galvanic effect.

Note that for a plane wave EM field, the anomalous electric field for near-surface heterogeneity is caused by galvanic charge buildup on the boundary of heterogeneity if its size is small relative to the penetration depth of the EM field (e.g., deGrootHedlin, 1991). This is also true for controlled-source EM responses if the Tx-Rx offset is large with respect to the penetration depth. In other words, the relative contribution of the galvanic effect to the near-surface response increases as the TxRx offset increases.

Both the amplitude and phase responses show that the effect of seafloor topography on the target signature is not significant in deepwater (Figure 5c and d). Figure 6 shows the electric field response of the bathymetric model with the reservoir, normalized by the response of the model without the reservoir. Such normalization appears to remove bathymetric effects, but note that the background resistivity is generally unknown for real
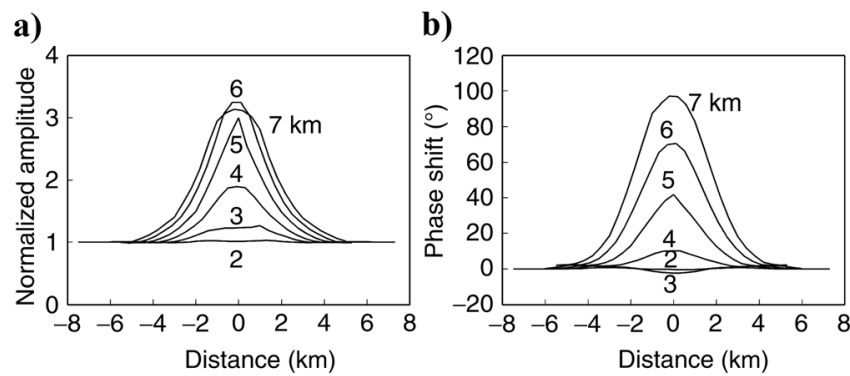

Figure 6. (a) Normalized amplitude and (b) phase difference for the electric field responses in Figure $5 \mathrm{c}$ and $d$. a)
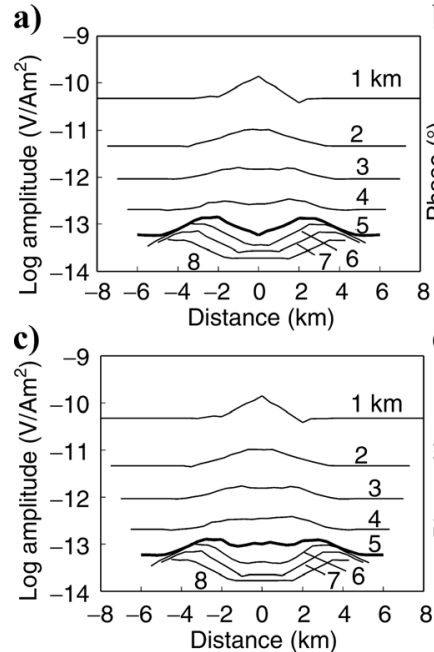

b)

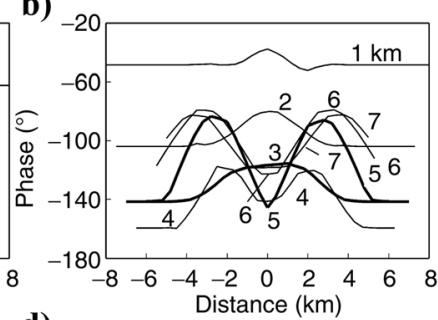

d)

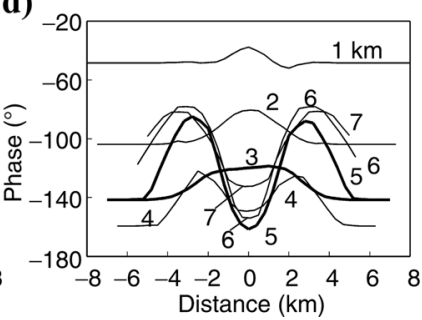

Figure 7. Inline horizontal electric field responses at selected Tx$\mathrm{Rx}$ offsets for the shallow-water models with and without the resistive reservoir ( $h=600 \mathrm{~m}$ in Figure 4). (a) Amplitude and (b) phase for the bathymetric model without the reservoir; (c) amplitude and (d) phase for the model with the reservoir. The source frequency is $0.25 \mathrm{~Hz}$. data and that the normalized response differs depending on the resistivity assumed in the bathymetric model.

\section{Electric field characteristics in shallow water}

Bathymetric effects in shallow-water CSEM data have been examined. For this simulation, the water depth at the bottom of the hill, $h$ is set to $600 \mathrm{~m}$, and so the shallowest depth is $200 \mathrm{~m}$ on the top of the ridge. Figure 7 shows the inline electric field responses of the models with and without the target reservoir. It is evident that the CSEM response is dominated by bathymetric anomalies in this shallow-water model. This result suggests that the bathymetric distortion becomes more significant as the water becomes shallower, and it will be practically impossible to identify the target response without knowing and incorporating the seafloor topography in the 3D CSEM model.

Although we are not able to quantify the relative contributions of galvanic and inductive effects, the galvanic effect appears not to be the dominant cause of the anomalous electric fields in the shallow water. Note that the galvanic effect for near-surface (or near-seafloor) heterogeneity causes no distortions in the phase response if the penetration depth is sufficiently large compared to the size of heterogeneity (e.g., deGroot-Hedlin, 1991). At shorter Tx-Rx offsets, the contribution of inductive effects to bathymetric distortions is more significant. The reason why the bathymetric distortions due to the induced currents are greater in the shallow water is that the bulk resistivity variations associated with seafloor topography are effectively more significant in the shallow water for a given Tx-Rx offset because bathymetry undulation is greater relative to the thickness of the water layer. At long offsets, the distortions are further complicated by the airwave signature, particularly in the phase response; at offsets longer than $5 \mathrm{~km}$, depressions around $x=0$ are accompanied by peaks on both sides in both the amplitude and phase response profiles. Note that the central depressions correspond to the Tx-Rx geometry where both the source and receiver are located at the deepest level, whereas the peaks correspond to where either the source or receiver is located at the shallowest level (i.e., over the top of the rise).

Although not shown here, a similar response pattern is seen if no topographic rise occurs (i.e., a flat seafloor), but the source and receiver are maintained at the same positions relative to the sea surface as in the ridge model. This means that this particular response pattern at longer offsets in the profiles is mainly determined by the Tx-Rx geometries relative to the sea surface rather than by the resistivity variations associated with the seafloor topographic changes, although the magnitude of the airwave signature is certainly affected by seabed resistivity (Andreis and MacGregor, 2008).

Target signatures can be seen clearly in the normalized electric field for the shallow-water reservoir model (Figure 8) as shown, although the magnitude is much smaller than that in the deepwater. The presence of a target generally increases the normalized amplitude at intermediate offsets, decreases it at long offsets, and moves the phase-shift curve downward. This general behavior is basically the same as that found for a flat-seafloor model (Sasaki and Meju, 2009). In addition to this general characteristic, the target response has a feature unique to the particular bathymetric model considered in this study. That is, a 
sharp peak occurs when the source and receiver are located on both the flanks of the hill, being accompanied by undershoots when the source and receiver straddle the bottom edge of the hill. This implies that the bathymetry and target are coupled to some degree.

\section{Applicability of the correction method}

The bathymetric correction technique was first applied to the deepwater CSEM responses presented above (Figure 5c and d). In Figure 9, the amplitude and phase responses corrected for bathymetric effects are compared to the responses of the corresponding flat-seafloor reservoir model. Corrections were made according to equation 2, assuming two different homogeneous resistivities (1 and $3 \Omega-m$ ) for the bathymetric model. For the true background resistivity case $(1 \Omega-\mathrm{m})$, the amplitude profile can be corrected by multiplying the normalized profile shown in Figure 6a with the theoretical response of the flat-seafloor homogeneous model; adding the phase response of the flat-seafloor homogeneous model to the phase shift profile shown in Figure 6b yields the corrected phase response. The corrected results for both resistivities compare favorably with the flat-seafloor model response. Further tests assuming background resistivities of 2 and $5 \Omega$-m gave virtually the same results as in Figure 9.

To see if the bathymetric correction still works when shallow heterogeneity is present, it was applied to the electric field response for a deepwater model that is the same as that shown in Figure 4 but includes a $2 \mathrm{D}$ anomalous body with a resistivity of $5 \Omega-\mathrm{m}$ at a burial depth of $80 \mathrm{~m}$ (outlined with the dashed parallelogram in Figure 4). Figure 10 shows comparisons of the corrected electric fields using background resistivities of 1 and $3 \Omega-\mathrm{m}$ and the response of the corresponding flat-seafloor model. The agreement is generally good. This experiment shows that the correction technique is effective in reducing bathymetric effects in deepwater regardless of the background resistivity chosen. Small discrepancies remaining, particularly in the phase profiles, are considered to be due to the difference in the Tx-Rx geometry in the bathymetric and flatseafloor models relative to the target reservoir and shallow body, but the magnitude of discrepancies is practically negligible.

Next, the correction procedure was tested on the electric field response for the shallow-water case (Figure $7 \mathrm{c}$ and d). Figure 11 shows comparisons of the corrected response obtained with the true background resistivity $(1 \Omega-\mathrm{m})$ and the response of the flatseafloor reservoir model. Unlike the deepwater case, the difference is rather significant, particularly in the phase response. When the bathymetric model with a resistivity of $3 \Omega-m$ is used in the correction, the resulting profile (not shown) departs further from the flat-seafloor model response. Such large differences are mainly caused by the difference in the Tx-Rx geometry relative to the sea surface rather than to the deepseated target; in other words, they are mainly due to the difference in the airwave responses in the two models. From this example, it becomes clear that the bathymetric and target responses are coupled through the airwave effect in shallow water and that the correction technique is not applicable over the Tx-Rx offset ranges at which the airwave signature occurs.
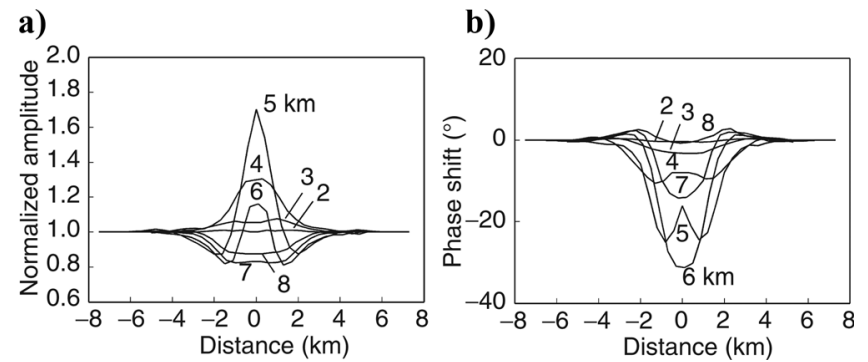

Figure 8. (a) Normalized amplitude and (b) phase difference for the electric field responses in Figure $7 \mathrm{c}$ and d.
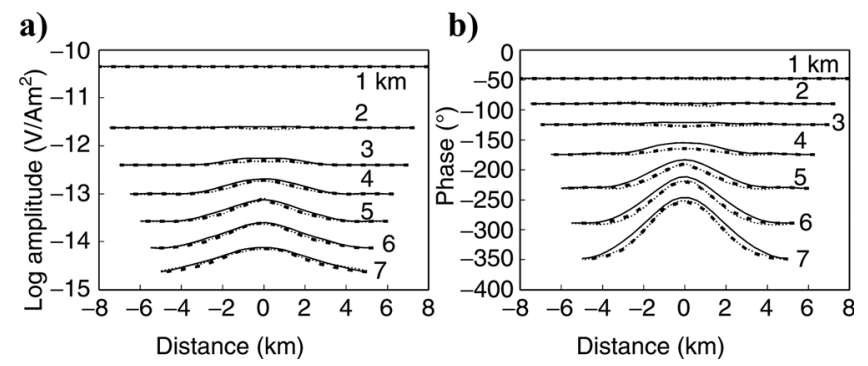

Figure 9. (a) Amplitude and (b) phase responses for the deepwater reservoir model, corrected for bathymetric effects using background resistivities of 1 and $3 \Omega-\mathrm{m}$ (dashed and dotted lines), and the corresponding responses of the flat-seafloor reservoir model (solid lines). The flat-seafloor model has a water layer of $2000 \mathrm{~m}$.

a)

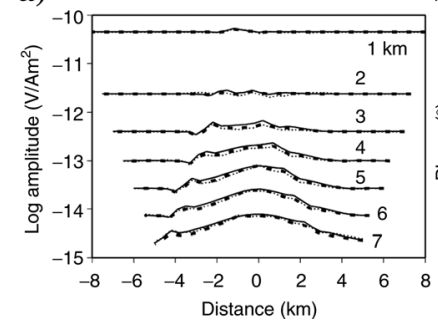

b)

Figure 10. (a) Amplitude and (b) phase responses for the deepwater model with the reservoir plus shallow heterogeneity, corrected for bathymetric effects using background resistivities of 1 and $3 \Omega-m$ (dashed and dotted lines), and the corresponding responses of the flat-seafloor model (solid lines). The flat-seafloor model has a water layer of $2000 \mathrm{~m}$. a)

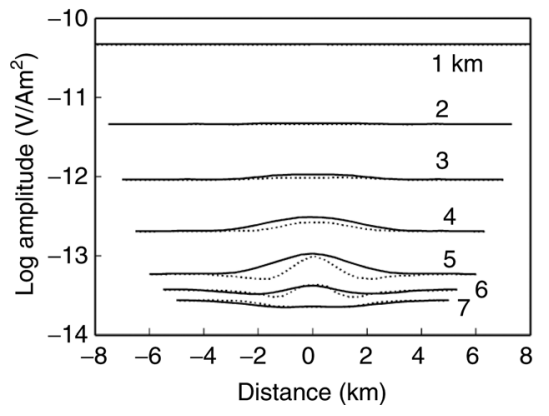

b)

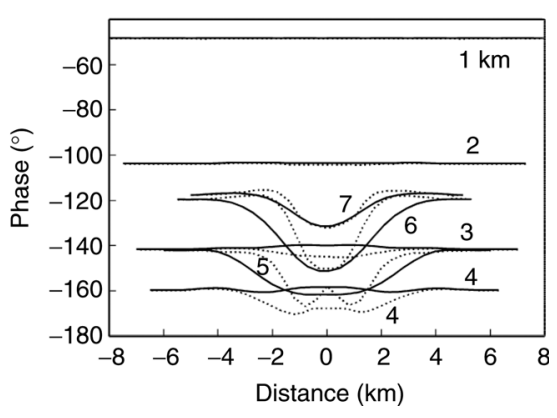

Figure 11. (a) Amplitude and (b) phase responses for the shallow-water reservoir model, corrected for bathymetric effects using a background resistivity of $1 \Omega$-m (dotted lines), and the corresponding responses of the flat-seafloor reservoir model (solid lines). The flat-seafloor model has a water layer of $600 \mathrm{~m}$. 


\section{EFFECT OF BATHYMETRY ON GAS HYDRATE RESPONSE}

The marine CSEM method also can be used to detect gas hydrates and has the potential to provide an assessment of hydrate content (Weitemeyer et al., 2006). Since gas hydrate accumulations occur at shallow depths below the seafloor, their detection by CSEM methods requires higher transmission frequencies and the collection of data at shorter Tx-Rx separations, as compared to deep hydrocarbon exploration such as the one

a)

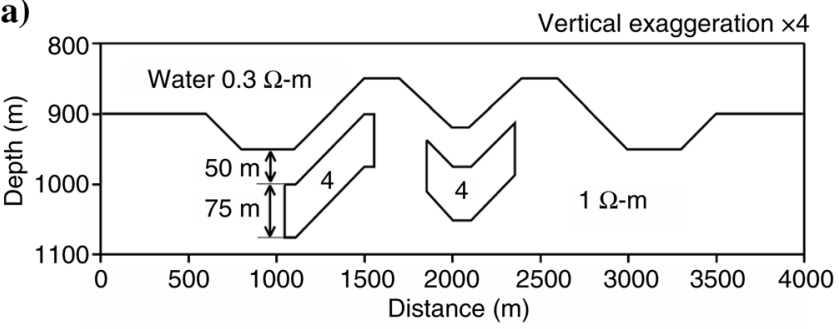

b)

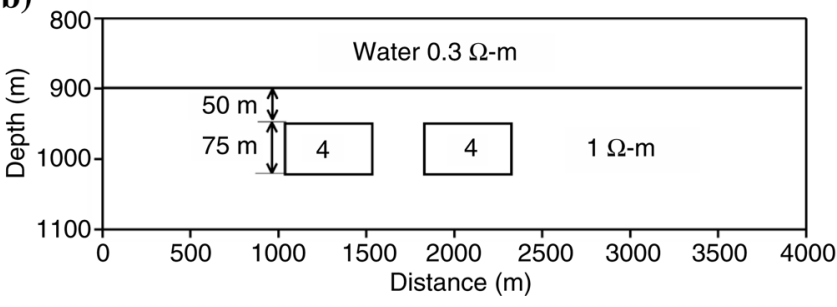

Figure 12. (a) $2 \mathrm{D}$ hydrate model with bathymetric variations and (b) the corresponding flat-seafloor model. The HED sources are located at a constant depth of $800 \mathrm{~m}$. a)

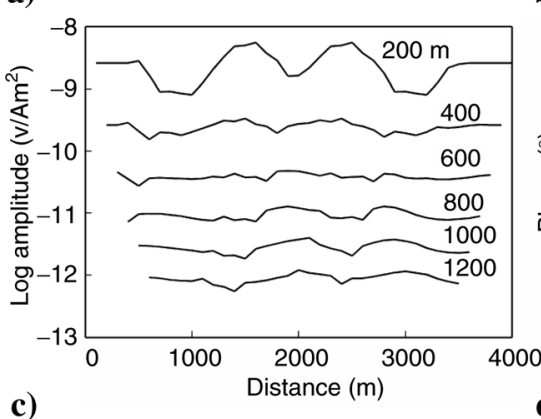

c)

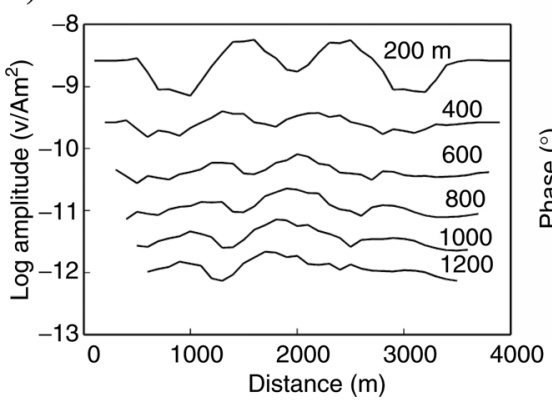

d)
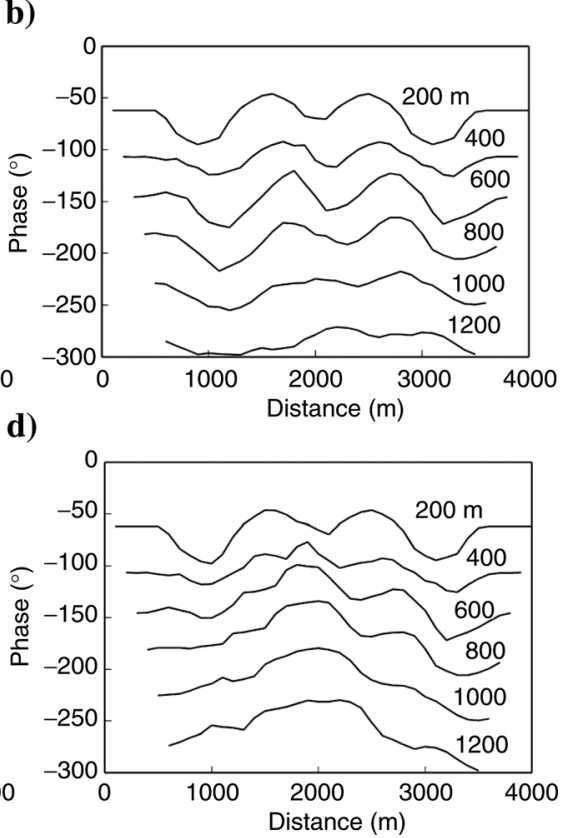

Figure 13. Inline horizontal electric field responses at selected Tx-Rx offsets for the bathymetric models with and without the hydrate patches. (a) Amplitude and (b) phase for the model with no hydrates; (c) amplitude and (d) phase for the model with the hydrates. The source frequency is $5 \mathrm{~Hz}$. considered previously in this study. To investigate the effect of bathymetry on gas-hydrate detection, I consider a 2D model with smaller-scale topographic variation (Figure 12a). Water depths range from 850 to $950 \mathrm{~m}$ over an undulating seafloor, and are $900 \mathrm{~m}$ for the flat seafloor. The model has two $4 \Omega-\mathrm{m}$ hydrate patches, each with a lateral extent of $500 \mathrm{~m}$ and a thickness of $75 \mathrm{~m}$, embedded in sediment with a background resistivity of $1 \Omega-\mathrm{m}$. The hydrate patches are spaced $300 \mathrm{~m}$ apart and are buried $50 \mathrm{~m}$ below the seafloor. The transmitted frequency is $5 \mathrm{~Hz}$. The inline horizontal electric field responses were simulated for seafloor receivers positioned every $50 \mathrm{~m}$ from $x=50$ to $4100 \mathrm{~m}$, using 40 HED sources of length $50 \mathrm{~m}$ spaced at $100 \mathrm{~m}$ intervals and located at a constant depth of $800 \mathrm{~m}$. The FD grid has $189 \times 78 \times 52$ cells, with a minimum cell size of $25 \times 50 \times 12.5 \mathrm{~m}$ in the region involving topographic undulation and a maximum size of $3.2 \times 3.2 \times 3.2 \mathrm{~km}$ at the bounding corners of the model.

\section{Electric field characteristics}

Figure 13 shows the inline horizontal electric field response plotted for selected Tx-Rx offsets (200 to $1200 \mathrm{~m}$ ) for the models with and without the hydrate bodies. The bathymetric distortions in the electric field amplitude and phase are seen to get smaller with increasing $\mathrm{Tx}-\mathrm{Rx}$ offsets and short-wavelength components in the amplitude profiles persist at all Tx-Rx offsets (Figure 13a and b). This characteristic is consistent with that of the deepwater model response (Figure 5), but the magnitudes of anomalous responses for the hydrate model are much larger because of the higher frequency and shorter Tx-Rx offset that are used. Thus, the bathymetric response appears to mask the hydrate signature, so it would be almost impossible to infer the hydrate response from Figure $13 \mathrm{c}$ and $\mathrm{d}$ without modeling the bathymetric responses.

To examine the hydrate signature, the electric field response of the hydrate model (Figure $13 \mathrm{c}$ and $\mathrm{d}$ ) was normalized by the response of the model with no hydrates (Figure 13a and b). The normalized profiles (Figure 14) show that at short offsets (400 and $600 \mathrm{~m}$ ), the peaks of signatures in both the amplitude and phase profiles correspond well to the centers of the hydrate patches, whereas at greater offsets $(1000$ and $1200 \mathrm{~m})$ the highest peaks occur over between the actual hydrate patches, indicating the importance of collecting data at different offsets (ranging from short to long) for accurate mapping of gas hydrates.

\section{Corrected electric field}

The bathymetric correction technique was applied to the hydrate model response in Figure $13 \mathrm{c}$ and $\mathrm{d}$. The corrected responses were obtained using two background resistivities of 1 and $3 \Omega-\mathrm{m}$. In Figure 15, the corrected profiles are compared to the response of the flatseafloor hydrate model shown in Figure $12 \mathrm{~b}$. Overall, the agreement is good. There are some noticeable discrepancies in the phase profiles at shorter Tx-Rx offsets for the case where a $3 \Omega$ - 
a)

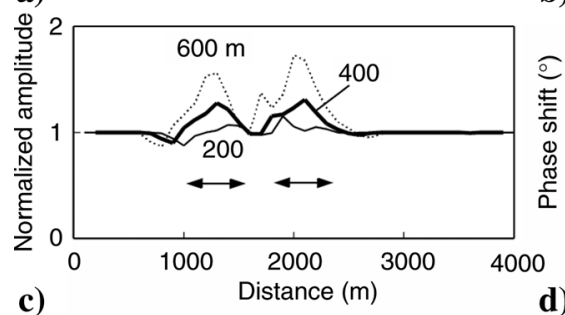

c)

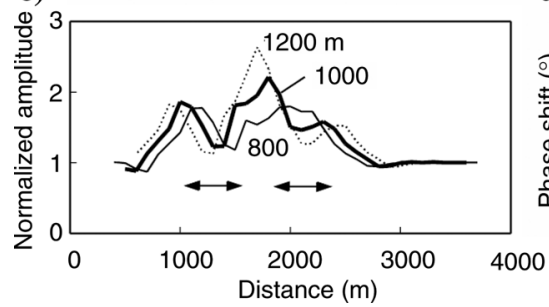

d)

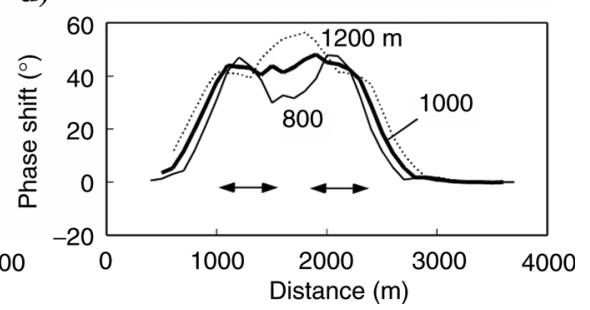

b)

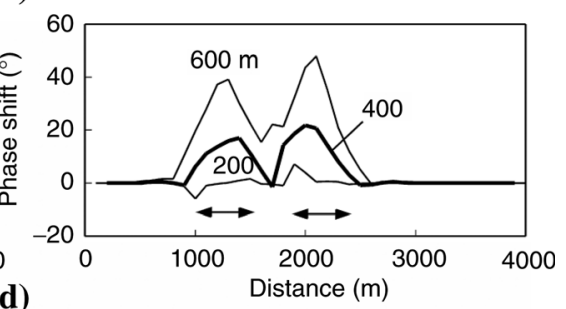

\section{.}

Figure 14. Normalized amplitude (left) and phase differences (right) for the electric field responses (Figure $13 \mathrm{c}$ and d). The profiles at shorter offsets are shown in (a, b), and those at longer offsets are in (c, d). The ranges over which the hydrate is present are indicated by the arrowed lines.

a)

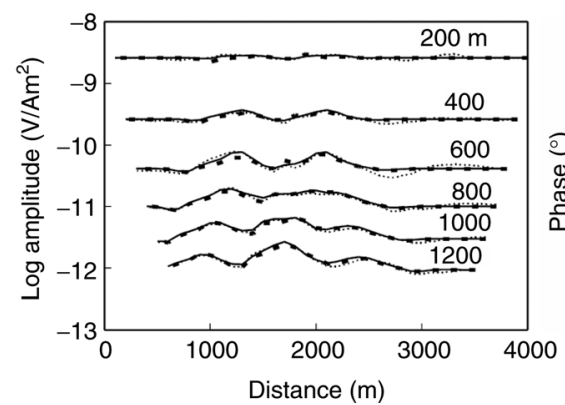


Commer, M., and G. A. Newman, 2007, 3D CSEM modeling and inversion for hydrocarbon reservoir mapping: The bathymetry problem: 77th Annual International Meeting, SEG, Expanded Abstracts, 452-456.

Constable, S., and L. J. Srnka, 2007, An introduction to marine controlledsource electromagnetic methods for hydrocarbon exploration: Geophysics, 72, no. 2, WA3-WA12.

Darnet, M., M. C. K. Choo, R.-E. Plessix, M. L. Rosenquist, K. Yip-Cheong, E. Sims, and J. W. K. Voon, 2007, Detecting hydrocarbon reservoirs from CSEM data in complex settings: Application to deepwater Sabah, Malaysia: Geophysics, 72, no. 2, WA97-WA103.

DeGroot-Hedlin, C., 1991, Removal of static shift in two dimensions by regularized inversion: Geophysics, 56, no. 12, 2102-2106.

Ellingsrud, S., T. Eidesmo, S. Johansen, M. C. Sinha, L. M. MacGregor, and S. Constable, 2002, Remote sensing of hydrocarbon layers by seabed logging (SBL): Results from a cruise offshore Angola: The Leading Edge, 21, no. 10, 972-982.

Endo, M., M. Cuma, and M. S. Zhdanov, 2008, Large-scale electromagnetic modeling for multiple inhomogeneous domains: 78th Annual International Meeting, SEG, Expanded Abstracts, 589-593.

Fox, R. C., G. W. Hohmann, T. J. Killpack, and L. Rijo, 1980, Topographic effects in resistivity and induced-polarization surveys: Geophysics, 45, no. 1, 75-93.
Hesthammer, J., A. Stefatos, M. Boulaenko, S. Fanavoll, and J. Danielsen, 2010, CSEM performance in light of well results: The Leading Edge, 29, no. 1, 34-41.

Hoversten, G. M., G. A., Newman, N. Geier, and G. Flanagan, 2006, 3D modeling of a deepwater EM exploration survey: Geophysics, 71, no. 5 , G239-G248.

Li, Y., and S. Constable, 2007, 2D marine controlled-source electromagnetic modeling: Part 2 - The effect of bathymetry: Geophysics, 72, no. 2, WA63-WA71.

Newman, G. A., and D. L. Alumbaugh, 1995, Frequency-domain modeling of airborne electromagnetic responses using staggered finite differences: Geophysical Prospecting, 43, no. 8, 1021-1042, doi:10.1111/ j.1365-2478.1995.tb00294.x.

Sasaki, Y., and M. A. Meju, 2009, Useful characteristics of shallow and deep marine CSEM responses inferred from 3D finite-difference modeling: Geophysics, 74, no. 5, F67-F76, doi:10.1190/1.3168616.

Um, E., 2005, On the physics of galvanic source electromagnetic geophysical methods for terrestrial and marine exploration: M.S. thesis, University of Wisconsin-Madison.

Weitemeyer, K., S. Constable, and K. Key, 2006, Marine EM techniques for gas-hydrate detection and hazard mitigation: The Leading Edge, $\mathbf{2 5}$ no. 5. 629-632, doi:10.1190/1.2202668. 